\title{
Memory enhancement induced by post-training intrabasolateral amygdala infusions of $\beta$-adrenergic or muscarinic agonists requires activation of dopamine receptors: Involvement of right, but not left, basolateral amygdala
}

\author{
Ryan T. LaLumiere ${ }^{1}$ and James L. McGaugh \\ Center for the Neurobiology of Learning and Memory and Department of Neurobiology and Behavior, University of California, \\ Irvine, California 92697, USA
}

\begin{abstract}
Previous findings indicate that the noradrenergic, dopaminergic, and cholinergic innervations of the basolateral amygdala (BLA) modulate memory consolidation. The current study investigated whether memory enhancement induced by post-training intra-BLA infusions of a $\beta$-adrenergic or muscarinic cholinergic agonist requires concurrent activation of dopamine (DA) receptors in the BLA. Rats with implanted BLA cannulae were trained on an inhibitory avoidance (IA) task and, $48 \mathrm{~h}$ later, tested for retention. Infusions of the $\beta$-adrenergic agonist clenbuterol into the right $\mathrm{BLA}$, but not the left, enhanced retention, and concurrent infusions of the nonspecific DA receptor antagonist cis-Flupenthixol (Flu) blocked the enhancement. Post-training infusions of the muscarinic agonist oxotremorine into the right BLA also enhanced retention, and concurrent infusions of Flu blocked this effect. Additional experiments investigated whether memory modulation was lateralized to the right BLA. Post-training DA infusions into the right BLA, but not the left, enhanced retention. Post-training infusions of lidocaine or muscimol, which impair retention when infused bilaterally, had no effect when infused unilaterally into either the right or left BLA. These findings, together with earlier work, suggest that the dopaminergic system in the BLA is critically involved in memory modulation induced by noradrenergic and cholinergic influences. Additionally, these findings indicate that the enhancement, but not impairment, of memory consolidation is lateralized to the right BLA.
\end{abstract}

Considerable evidence indicates that the dopaminergic, noradrenergic, and muscarinic cholinergic systems in the basolateral amygdala (BLA) play important roles modulating memory consolidation. Post-training intra-BLA infusions of noradrenergic agonists, norepinephrine, dopamine (DA), or muscarinic cholinergic agonists enhance retention of training in a variety of tasks (Introini-Collison et al. 1996; Hatfield and McGaugh 1999; LaLumiere et al. 2003, 2004; Power et al. 2003a; McGaugh 2004). Moreover, these systems are critical for memory modulation induced by other neurotransmitters or hormones. Post-training intra-BLA infusions of $\beta$-adrenoceptor antagonists block the memory-enhancing effects of opioid-peptidergic antagonists, glucocorticoid agonists, and DA (McGaugh et al. 1988; Quirarte et al. 1997; LaLumiere et al. 2004). Similarly, post-training intraBLA infusions of muscarinic cholinergic antagonists block the memory-enhancing effects of glucocorticoid agonists, $\beta$-adrenergic agonists, and DA (Introini-Collison et al. 1996; Power et al. 2000; LaLumiere et al. 2004).

Studies of the effects of DA receptor antagonists provide additional evidence suggesting that the dopaminergic system in the BLA is also critically involved in memory modulation. Post-training intra-BLA infusions of a D1 or D2 receptor antagonist impair retention for inhibitory avoidance (IA) training (LaLumiere et al. 2004), and post-training intra-BLA infusions

\footnotetext{
${ }^{1}$ Corresponding author.

E-mail lalumie@musc.edu; fax (843) 792-4423.

Article and publication are at http://www.learnmem.org/cgi/doi/10.1101/ Im.97405.
}

of the nonspecific DA receptor antagonist cis-Flupenthixol (Flu) block the memory enhancement induced by intranucleus accumbens shell infusions of DA (LaLumiere et al. 2005). Electrophysiological evidence indicates that activation of DA receptors within the BLA is critical for the suppression of inhibitory input from the medial prefrontal cortex and potentiation of excitatory input from the sensory cortices (Rosenkranz and Grace 2002). Moreover, it appears that D1 and D2 receptors regulate lateral amygdala (part of the BLA) interneuron activity, and that application of DA to lateral amygdala slices induces endogenous activity oscillations in interneurons that may be critical to synchronous firing of the projection neurons (Loretan et al. 2004). Such findings suggest DA receptor activation in the BLA critically influences BLA projection neuron activity. However, it is not known whether the noradrenergic and muscarinic cholinergic systems in the BLA require concurrent DA receptor activation to modulate memory consolidation. The current experiments investigated this issue.

Previous evidence from studies using reversible inactivation of the amygdala suggested that the right amygdala may be differentially involved in influencing memory consolidation (Coleman-Mesches and McGaugh 1995; Coleman-Mesches et al. 1996). Additionally, Baker and Kim (2004) reported that unilateral lesions of the right amygdala induced 6-h post-training were as effective as bilateral lesions in impairing retention of fear conditioning. Thus, the current experiments also examined further the issue of whether BLA-influenced modulation of memory consolidation is lateralized. 


\section{Results}

\section{Histology}

Figure $1 \mathrm{~A}$ shows a diagram of the BLA and Figure $1 \mathrm{~B}$ shows a representative photomicrograph of a cannula tip dorsal to the BLA and a needle track terminating in the BLA. The final analysis included only rats whose needle tracks were located in the BLA and had no lesions around the track. In Experiment 1, 190 rats had correct unilateral infusion needle placements (of 215). In Experiment 2, 184 rats had correct unilateral infusion needle placements (of 196). Figure 1C shows representative examples of needle infusion termination sites of 60 randomly selected rats (30 with left-side and 30 with right-side BLA infusions) included in the final analysis. Individual group $n$ 's are indicated in the figure legends. A $t$-test revealed no significant difference between the $\mathrm{A} / \mathrm{P}$ coordinates, in regard to Bregma, of termination sites in the left BLA (mean: $-2.75 \mathrm{~mm} \pm 0.061$ ) and those of termination sites in the right BLA (mean: $-2.72 \mathrm{~mm} \pm 0.062 ; t_{(58)}=0.305$, $P>0.05)$.

\section{Experiment 1}

The retention latencies of rats given immediate post-training intra-BLA infusions of Clen into the right or left BLA or the Clen/ Flu combination into the right BLA are shown in Figure 2. Figure $2 \mathrm{~A}$ shows the retention latencies of rats given infusions of the saline-vehicle or Clen into the left BLA. A one-way ANOVA revealed no effect $\left(F_{(3,42)}=0.314, P>0.05\right)$. Figure $2 \mathrm{~B}$ shows the retention latencies of rats given infusions of the saline-vehicle or Clen into the right BLA. A one-way ANOVA revealed a significant effect $\left(F_{(3,35)}=7.315, P<0.001\right)$. The latencies of rats given the 1 or 10-ng dose of Clen were significantly higher than those of rats given the vehicle or $100-n g$ dose of Clen $(P<0.01)$. The latencies of rats given the 100-ng dose of Clen were not significantly different from those of rats given the saline-vehicle $(P>0.05)$. Figure $2 \mathrm{C}$ shows the retention latencies of rats given infusions of Flu alone or the Clen/Flu combination. A one-way ANOVA revealed no effect $\left(F_{(3,28)}=0.008, P>0.05\right)$.

The retention latencies of rats given immediate posttraining infusions of Oxo or the Oxo/Flu combination into the
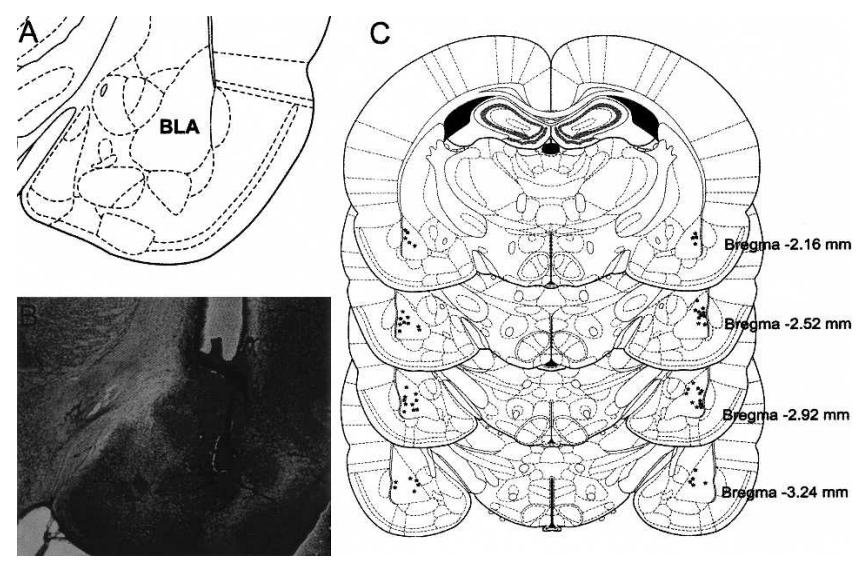

Figure 1. (A) Diagram of rat BLA and adjacent structures (Paxinos and Watson 2005; $2.8 \mathrm{~mm}$ posterior to Bregma). (B) Representative photomicrograph of needle track terminating in the BLA. Only data from animals that had needle tracks terminating in the BLA and had no lesions in the surrounding BLA tissue were included in the analyses. (C) Diagrams of rat brain sections (Paxinos and Watson 2005) $(2.16 \mathrm{~mm}, 2.52 \mathrm{~mm}, 2.92$ $\mathrm{mm}$, and $3.24 \mathrm{~mm}$ posterior to Bregma) showing 60 infusion needle termination sites (30 on each side) randomly selected from rats included in the final analysis. All diagrams of rat brain sections were adapted with permission from Elsevier (c) 2005, Paxinos and Watson (2005).

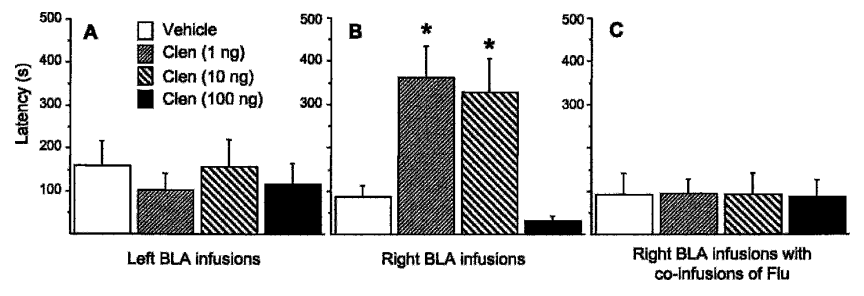

Figure 2. Retention of rats that received immediate post-training intraBLA infusions of Clen into the left or right BLA and infusions of the Clen/Flu combination into the right BLA. $(A)$ Mean latencies, in seconds, ( \pm SEM) of rats given Clen infusions into the left BLA to enter the shock compartment during the retention test. Groups (from left to right): Vehicle-controls (white bar, $n=16$ ), 1 ng Clen (narrow-hatched bar, $n=15), 10 \mathrm{ng}$ Clen (wide-hatched bar, $n=8$ ), $100 \mathrm{ng}$ Clen (black bar, $n=7)$. (B) Mean latencies, in seconds, ( \pm SEM) of rats given Clen infusions into the right BLA to enter the shock compartment during the retention test. Groups (from left to right): Vehicle-controls (white bar, $n=10), 1 \mathrm{ng}$ Clen (narrow-hatched bar, $n=10), 10 \mathrm{ng}$ Clen (widehatched bar, $n=11), 100$ ng Clen (black bar, $n=8)$. $\left(^{*}\right) P<0.01 \mathrm{com}$ pared with vehicle-control and 100-ng infusions into right BLA. (C) Mean latencies, in seconds, ( \pm SEM) of rats given infusions of the Clen/Flu combination into the right BLA to enter the shock compartment during the retention test. Groups (from left to right): $10 \mu \mathrm{g} \mathrm{Flu} \mathrm{(white} \mathrm{bar,} n=8$ ), Flu/Clen (1 ng) (narrow-hatched bar, $n=9)$, Flu/Clen (10 ng) (widehatched bar, $n=7)$, Flu/Clen (100 ng) (black bar, $n=8)$.

right BLA are shown in Figure 3. Figure 3A shows the retention latencies of rats given infusions of saline-vehicle or Oxo. A oneway ANOVA revealed a significant effect $\left(F_{(3,35)}=4.646\right.$, $P<0.01)$. The latencies of rats given the 10 -ng dose of Oxo were significantly higher than those of rats given the vehicle or 100-ng dose of Oxo $(P<0.005)$, but were not significantly different from those of rats given the 1-ng dose of Oxo. The latencies of rats given the 1- or 100-ng dose of Oxo were not significantly different from each other or those of rats given the saline-vehicle $(P>0.05)$. Figure 3B shows the retention latencies of rats given infusions of Flu alone or the Oxo/Flu combination. A one-way ANOVA revealed no effect $\left(F_{(3,30)}=0.137, P>0.05\right)$.

\section{Experiment 2}

The retention latencies of rats given immediate post-training intra-BLA infusions of DA into the left or right BLA are shown in Figure 4 . Figure $4 \mathrm{~A}$ shows the retention latencies of rats given infusions of saline-vehicle or DA into the left BLA. A one-way ANOVA revealed no effect $\left(F_{(3,37)}=0.069, P>0.05\right)$. Figure $4 \mathrm{~B}$ shows the retention latencies of rats given infusions of salinevehicle or DA into the right BLA. A one-way ANOVA revealed a significant effect $\left(F_{(3,40)}=3.033, P<0.05\right)$. The latencies of rats given the 3 - or $10-\mu$ g dose of DA were significantly higher than those of rats given the vehicle $(P<0.05$ and $P<0.01$, respectively).

The retention latencies of rats given immediate posttraining intra-BLA infusions of Musc or lidocaine are shown in Figure 5. Figure $5 \mathrm{~A}$ shows the retention latencies of rats given infusions of saline-vehicle or Musc into the left or right BLA. A two-way ANOVA revealed no main effects or interaction ( $P>0.05$ for all comparisons). Figure 5B shows the retention latencies of rats given infusions of saline-vehicle or lidocaine into the left or right BLA. A two-way ANOVA revealed no main effects or interaction ( $P>0.05$ for all comparisons).

\section{Discussion}

\section{BLA-induced memory modulation requires DA receptor activation}

The results of Experiment 1 indicate that post-training right-side, but not left-side, intra-BLA infusions of Clen enhanced retention. 


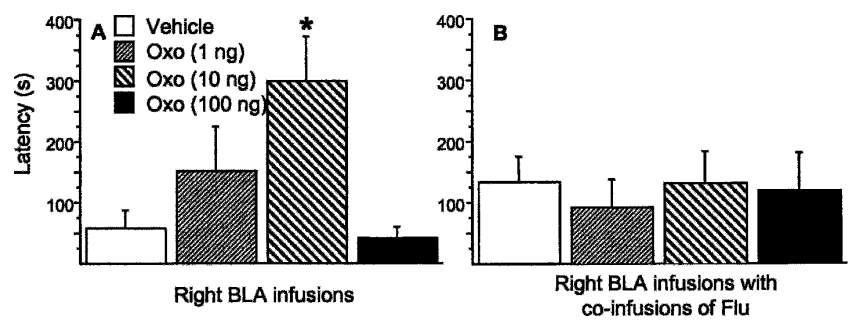

Figure 3. Retention of rats that received immediate post-training intraBLA infusions of Oxo or the Oxo/Flu combination into the right BLA. $(A)$ Mean latencies, in seconds, ( \pm SEM) of rats given Oxo infusions into the right BLA to enter the shock compartment during the retention test. Groups (from left to right): Vehicle-controls (white bar, $n=10$ ), 1 ng Oxo (narrow-hatched bar, $n=9$ ), 10 ng Oxo (wide-hatched bar, $n=11$ ), and $100 \mathrm{ng}$ Oxo (black bar, $n=9$ ). ( $\left.{ }^{*}\right) P<0.005$ compared with vehiclecontrols and 100-ng infusions. (B) Mean latencies, in seconds, ( \pm SEM) of rats given infusions of the Oxo/Flu combination into the right BLA to enter the shock compartment during the retention test. Groups (from left to right): $10 \mu \mathrm{g}$ Flu (white bar, $n=9$ ), Flu/Oxo $(1 \mathrm{ng})$ (narrow-hatched bar, $n=8)$, Flu/Oxo (10 ng) (wide-hatched bar, $n=9)$, and Flu/Oxo (100 ng) (black bar, $n=8$ ).

Concurrent infusions of Flu blocked this enhancement. The results of Experiment 1 also indicate that post-training right-side intra-BLA infusions of Oxo enhanced retention and that concurrent intra-BLA infusions of Flu blocked this enhancement. As all infusions in Experiments 1 and 2 were administered immediately after training, the animals were trained under drug-free conditions and, therefore, the drugs did not affect the animals' performance during training. These findings strongly suggest that activation of DA receptors in the BLA is critical for memory modulation by the noradrenergic and cholinergic systems in the BLA.

Evidence suggests that the mnemonic effects of particular BLA systems depend on concurrent activation of receptors for other systems. Previous studies indicate that the memorymodulating effects of drugs affecting the opioid-peptidergic or glucocorticoid systems require $\beta$-adrenoceptor activation in the BLA and that the memory-modulating effects of drugs affecting the glucocorticoid or noradrenergic systems require muscarinic receptor activation in the BLA (McGaugh et al. 1988; IntroiniCollison et al. 1996; Quirarte et al. 1997; Power et al. 2000). Such findings suggested that there is a sequential "order" of effects of these systems, and the muscarinic receptors appeared to be the most "downstream" system in the BLA in influencing memory consolidation. However, the present findings clearly suggest that the noradrenergic and muscarinic systems are not "downstream" from the DA system. As we previously found that either a $\beta$-adrenoceptor antagonist or muscarinic antagonist infused into the BLA post-training blocks the memory enhancement induced by concurrent DA infusions (LaLumiere et al. 2004), the memorymodulating effects of the muscarinic and DA systems appear to have a corequirement for activation of the other systems' receptors.

Such findings suggest that the DA, noradrenergic, and muscarinic systems within the BLA are all critical for memory modulation. However, as previous evidence indicates that intraamygdala infusions of $\beta$-adrenoceptor antagonists do not block the memory enhancement induced by concurrent infusions of muscarinic agonists (Introini-Collison et al. 1996), it is not clear why the DA, but not the muscarinic, system in the BLA requires $\beta$-adrenoceptor activation. One possibility is that the DA system may not completely depend on the noradrenergic and/or muscarinic systems. In the present experiments, three doses (including a high ineffective dose) of Clen and Oxo were used. As our previous experiment (LaLumiere et al. 2004) used only a single memory-enhancing dose of DA, it is possible that, in that experi- ment, the infusions of the $\beta$-adrenergic and muscarinic antagonists shifted the dose-response curve for DA-induced memory enhancement.

The current findings suggesting a critical role for BLA DA receptors during memory modulation are consistent with findings from other studies. Activation of BLA D1 and D2 receptors is critical for overall BLA activation (Rosenkranz and Grace 2002). Recent findings suggest that D1 receptor activation on BLA interneurons and projection neurons increases neuronal excitability through modulation of the slowly inactivating, outwardrectifying potassium current (Kroner et al. 2005). In contrast, D2 receptor activation increases the neuronal input resistance of projection neurons (Kroner et al. 2005). Other evidence suggests that D1 and D2 receptors regulate lateral amygdala (part of the BLA) interneuron activity and that application of DA to lateral amygdala slices induces endogenous activity oscillations in lateral amygdala interneurons that may be critical to synchronous firing of projection neurons (Loretan et al. 2004). Increases in such projection neuron synchronicity are associated with tone fear conditioning and may be involved in the modulation of memory via BLA projections to the cortex (Pare and Collins 2000).

The evidence that the noradrenergic and muscarinic systems in the BLA depend on activation of the DA system for memory modulation suggests that activation of $\beta$-adrenergic, muscarinic, and DA receptors produces downstream effects that "interact" with each other in BLA neurons. Although the $\beta$-adrenergic and glucocorticoid systems interact at the level of the cAMP-PKA pathway in BLA neurons (Roozendaal et al. 2002), it does not appear that activation of $\beta$-adrenergic, muscarinic, and DA receptors leads to a common intracellular pathway. Activation of muscarinic receptors on BLA projection neurons influences membrane-bound ion channels, changing their sodium and potassium conductance and, thus, depolarizing the neurons (Yajeya et al. 1999). Although activation of DA receptors in other brain regions including the striatum works through changes in adenylyl cyclase activity (Kebabian et al. 1972), recent evidence suggests that activation of D1 receptors in the BLA does not affect levels of cAMP, phospholipase C, or intracellular calcium (Leonard et al. 2003; Loretan et al. 2004). Instead, it appears that D1 receptors work through Src, a family of nonreceptor tyrosine kinases, to produce activity changes in BLA interneurons (Loretan et al. 2004), though it is unknown whether D1 receptor activation in projection neurons modulates Src or by what mechanism D2 receptor activation influences input resistance. Therefore, as

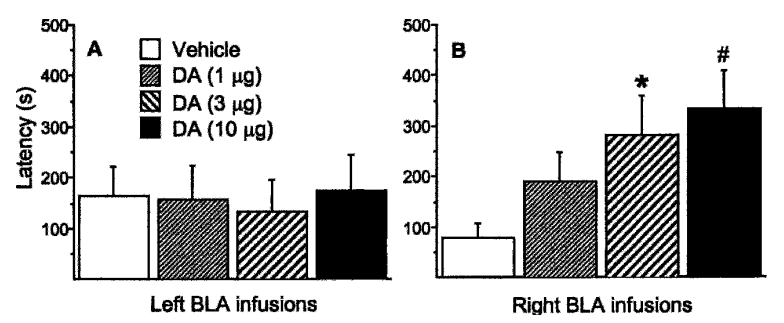

Figure 4. Retention of rats that received immediate post-training DA infusions into the left or right BLA. $(A)$ Mean latencies, in seconds, $( \pm S E M)$ of rats given DA infusions into the left BLA to enter the shock compartment during the retention test. Groups (from left to right): Vehicle-controls (white bar, $n=10$ ), $1 \mu \mathrm{g}$ DA (narrow-hatched bar, $n=11$ ), $3 \mu \mathrm{g}$ DA (wide-hatched bar, $n=9$ ), and $10 \mu \mathrm{g}$ DA (black bar, $n=11$ ). (B) Mean latencies, in seconds, ( \pm SEM) of rats given DA infusions into the right BLA to enter the shock compartment during the retention test. Groups (from left to right): Vehicle-controls (white bar, $n=10), 1 \mu \mathrm{g}$ DA (narrow-hatched bar, $n=12$ ), $3 \mu \mathrm{g}$ DA (wide-hatched bar, $n=11$ ), and $10 \mu \mathrm{g}$ DA (black bar, $n=11$ ). $\left(^{*}\right) P<0.05$ and (\#) $P<0.01$ compared with vehicle-controls. 

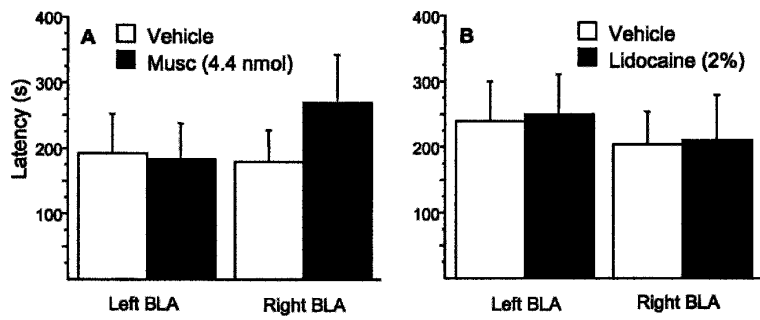

Figure 5. Retention of rats that received immediate post-training Musc or lidocaine infusions into the left or right BLA. $(A)$ Mean latencies, in seconds, $( \pm$ SEM) of rats given Musc infusions into the left or right BLA to enter the shock compartment during the retention test. Groups (from left to right): Vehicle-controls/Left side (white bar, $n=14$ ), $4.4 \mathrm{nmol}$ Musc/ Left side (black bar, $n=13$ ), Vehicle-controls/Right side (white, $n=12$ ), and $4.4 \mathrm{nmol}$ Musc/Right side (black bar, $n=12$ ). (B) Mean latencies, in seconds, $( \pm$ SEM) of rats given lidocaine $(2 \%)$ infusions into the left or right BLA to enter the shock compartment during the retention test. Groups (from left to right): Vehicle-controls/Left side (white bar, $n=13$ ), lidocaine/Left side (black bar, $n=13$ ), vehicle-controls/Left side (white bar, $n=12$ ), and lidocaine/Right side (black bar, $n=10$ ).

there does not appear to be a single intracellular cascade that serves as a final common pathway, it seems likely that each system, and perhaps in particular the muscarinic and DA systems, are involved in regulating different, yet critical, components of BLA network activity, activation of BLA projection neurons, and the subsequent modulation of memory consolidation.

Lateralization of memory enhancement to the rightBLA The present findings indicate that right side, but not left side, intra-BLA infusions of Clen enhanced retention. Similarly, the results of Experiment 2 indicate that right-side, but not left-side, intra-BLA infusions of DA enhanced retention. The results of Experiment 2 also indicate that unilateral intra-BLA infusions of Musc or lidocaine did not affect retention when infused into either the right or left BLA. The lack of effect on retention by Musc and lidocaine is not due to a dosage issue, as previous findings indicate that bilateral intra-amygdala/BLA infusions of the doses used in the current study impair retention (ColemanMesches and McGaugh 1995; Wilensky et al. 2000). These findings suggest that the right BLA, but not the left, is involved in the enhancing modulation of memory consolidation induced by intra-BLA infusions and that either BLA alone, intact, and unmanipulated, is sufficient for normal retention.

In light of that conclusion, it is not clear why the infusions of memory-enhancing drugs into the left BLA have no effect. In fact, the magnitudes of memory enhancement found in the current study with right-side intra-BLA infusions of Clen and DA are comparable to those found with bilateral infusions (Ferry and McGaugh 1999; LaLumiere et al. 2004), suggesting that drug infusions into the right BLA are sufficient to produce maximal memory enhancement. The use of many drug doses, including a high ineffective dose in the Clen experiment, suggests that the lack of effect with left BLA infusions is not simply a dosage issue.

Earlier evidence also indicated a greater involvement of the right BLA in memory and emotion (Coleman-Mesches and McGaugh 1995; Coleman-Mesches et al. 1996). Additionally, lesions of the right BLA induced 6-h post-training induced greater retention impairment for contextual fear conditioning than did lesions of the left BLA (Baker and Kim 2004). Other previous findings indicated that kindling of the right BLA in rats is anxiogenic, whereas kindling of the left BLA is anxiolytic (Adamec and Morgan 1994) and that higher serotonin levels in the right amygdala correlate with greater anxiety in an elevated plus maze (Andersen and Teicher 1999). Recent studies indicate that activity of the right amygdala in human males and the left amygdala in human females during encoding of emotionally arousing material correlates with long-term retention for the material (Cahill et al. 2001, 2004; Canli et al. 2002). Although the current findings did not use female rats, they are consistent with a greater consolidation role for the right amygdala in males.

The mechanism underlying the present lateralization findings is not clear. Evidence suggests that there are hemispheric asymmetries in the concentration of various neurochemicals including DA and norepinephrine (Rosen et al. 1984), though these studies have not examined the amygdala for differences. However, as other studies using lesions or inactivations of the amygdala also find greater involvement of the right amygdala, it seems likely that the mechanism for this lateralization is broader than simply differences in neurochemical or receptor distributions. Stressful or emotionally arousing stimuli may activate brainstem structures in a lateralized manner or, alternatively, downstream structures involved in mediating emotional arousal, such as the BLA, may process the information differently on the left and right sides of the brain. In addition, the BLA may differentially influence structures that receive BLA projections.

\section{Conclusions}

The present findings, together with those of previous studies, strongly indicate that activation of DA receptors in the BLA is critical for BLA-induced memory modulation. Specifically, memory enhancement by noradrenergic or muscarinic agonist infusions into the BLA requires DA receptor activation. Most likely, the requirement of these systems for DA receptor activation to influence memory does not reflect a final common intracellular pathway, such as a protein kinase cascade. Instead, evidence suggests that each system has its own critical effects on overall network activity within the BLA. In addition, the present findings suggest that the right BLA plays a more important role than the left BLA in modulating memory consolidation.

\section{Materials and Methods}

\section{Subjects}

Male Sprague-Dawley rats ( $\sim 300$ grams at the time of surgery, Charles River; $n=411$ ) were used in this study. They were individually housed, maintained in a temperature-controlled environment $\left(22^{\circ} \mathrm{C}\right)$ on a 12 -h light/12-h dark cycle (lights on at 07:00 h) with food and water ad libitum and given 7-8 d to acclimatize to the vivarium before undergoing surgery. Behavioral procedures began 7-9 d after surgeries. All methods used complied with NIH guidelines for care of laboratory animals and were approved by the UC Irvine Institutional Animal Care and Use Committee.

\section{Surgery}

The rats were anesthetized with sodium pentobarbital $(50 \mathrm{mg} / \mathrm{kg}$, i.p.) and given atropine sulfate $(0.1 \mathrm{mg}$, i.p.) to prevent respiratory congestion as well as $3.0 \mathrm{~mL}$ of saline (s.c.) to prevent dehydration during surgery. Supplemental doses of sodium pentobarbital were given as needed during surgery. The rats were then placed in a small animal stereotaxic instrument (Kopf Instruments). Two surgical screws were implanted into the skull as anchors and guide cannulae were implanted unilaterally in the left or right hemisphere, aimed at the basolateral amygdala (BLA, $2.8 \mathrm{~mm}$ posterior and $5.0 \mathrm{~mm}$ lateral to Bregma and $6.5 \mathrm{~mm}$ ventral to the skull surface) (Paxinos and Watson 2005). The nose bar was maintained at $-3.5 \mathrm{~mm}$ relative to the interaural line. The guide cannulae were constructed of 23-gauge stainless-steel tubing cut to a length of $15.00( \pm 0.02) \mathrm{mm}$. The cannulae and the screws were affixed to the skull with dental cement. Insect pins (15-mm long 00 insect dissection pins) were inserted into 
the cannulae to maintain patency and were removed only for the infusions. After the surgery, the rats were retained in an incubation chamber until they awoke. They were then returned to their home cages and checked on the days following surgery to ensure that their wounds healed.

\section{Behavioral procedures}

The rats were trained on a step-through inhibitory avoidance (IA) task. The IA apparatus was a 91-cm long, 20-cm deep troughshaped alley. The alley was divided into two compartments, a safe compartment (31-cm long) constructed of plastic and illuminated by a tensor lamp and a darkened shock compartment (60-cm long) with walls and floors constructed of stainless steel. The compartments were separated by a stainless-steel door that retracted into the floor. The floor of the dark compartment was connected to an AC shock generator (Lafayette Instruments) controlled by a timer.

Each rat was handled 1 min per day for $3 \mathrm{~d}$ prior to the start of training. All training and testing occurred between 10:00 a.m. and 4:30 p.m. Immediately after the training and testing of each animal, the apparatus was cleaned with a $20 \%$ ethanol solution. On the training day, each rat was placed into the safe compartment, with the door retracted below the floor, and permitted to explore the apparatus freely. After the rat entered the dark compartment, the door was raised to prevent it from re-entering the safe compartment. When the rat reached the end of the dark compartment, it received a single inescapable footshock. After the rat was removed from the apparatus, it received its appropriate intra-BLA drug infusions and was replaced in its home cage.

Retention was tested $48 \mathrm{~h}$ later. Each animal was placed into the safe compartment and permitted to explore the apparatus freely. The animal's initial latency to step into the dark compartment with all four paws was measured in seconds (sec) with a maximum latency of $600 \mathrm{sec}$.

\section{Drugs and drug infusion procedures}

Clenbuterol (Clen), cis-Flupenthixol (Flu), oxotremorine (Oxo), dopamine (DA), muscimol (Musc), and lidocaine were obtained from Sigma. All drugs were dissolved in saline (0.9\%). Doses of Clen and Oxo $(1,10$, and $100 \mathrm{ng})$ were chosen based on previous evidence suggesting that this range contained a dose(s) effective at enhancing memory when infused bilaterally into the BLA and to allow for any upward shift in the dose-response curve (Introini-Collison et al. 1996; Power et al. 2003b). A single dose of Flu was used $(10.0 \mu \mathrm{g})$ as previous findings suggest that this dose is sufficient to block memory modulation (LaLumiere et al. 2005). The doses of DA (1, 3, and $10 \mu \mathrm{g})$, Musc (4.4 $\mathrm{nmol})$, and lidocaine $(2 \%)$ were chosen based on previous evidence of their effectiveness in enhancing (DA) or impairing (Musc and lidocaine) memory when infused bilaterally into the amygdala/BLA (Coleman-Mesches and McGaugh 1995; Wilensky et al. 2000; LaLumiere et al. 2004).

To infuse the drug or vehicle into the BLA, PE-20 polyethylene tubing was connected to a $10-\mu \mathrm{L}$ Hamilton syringe and a 30-gauge dental needle was cemented to the other end of the tubing. The infusion needle was then bent to a length of $17 \mathrm{~mm}$ so that it extended $2 \mathrm{~mm}$ beyond the end of the guide cannula into the BLA. The tubing was first filled with distilled water. A small air bubble, followed by the drug or vehicle, was then pulled in. The Hamilton syringe was driven by an automated syringe pump (Sage Instruments) at the rate of $0.38 \mu \mathrm{L} / \mathrm{min}$. To perform the infusion, the pins were removed from the cannulae and the infusion needles were inserted. The syringe pump was turned on for $32 \mathrm{sec}$ in order to give an infusion volume of $0.2 \mu \mathrm{L}$ into the BLA. The needles were then left in place for an additional $35 \mathrm{sec}$ to allow the solution to diffuse. Immediately following the infusions, the animals were returned to their home cages.

\section{Experiment 1}

Rats were trained on the IA task (footshock: $0.45 \mathrm{~mA}, 1 \mathrm{sec}$ ) and given immediate post-training infusions of Clen or vehicle (sa- line) into either the left or right BLA. As Clen infusions into the right BLA, but not the left BLA, enhanced retention (Fig. 2A,B), the remainder of Experiment 1 used rats with unilateral, rightside cannulas aimed at the BLA. To determine whether DA receptor activation in the BLA was necessary for Clen infusions to enhance memory, other rats received right-side infusions of Clen or vehicle with a concurrent infusion of Flu. To determine whether Oxo-induced memory enhancement requires DA receptor activation in the BLA, other rats were given immediate posttraining right-side infusions of Oxo or vehicle (saline) either alone or with concurrent infusions of Flu.

\section{Experiment 2}

As the findings from Experiment 1 suggested that only righthemisphere intra-BLA infusions of Clen enhance retention, Experiment 2 investigated this issue further. To determine whether other neuromodulatory systems in the BLA also produced a lateralization effect, rats with either left or right-BLA cannulae were trained on the IA task (footshock: $0.45 \mathrm{~mA}, 1 \mathrm{sec}$ ) and received immediate post-training infusions of DA or vehicle (saline). To determine whether these lateralization effects could be obtained with memory-impairing drug infusions, other rats were trained on the IA task $(0.65 \mathrm{~mA}, 1 \mathrm{sec})$ and given post-training infusions of Musc, lidocaine, or vehicle (saline).

\section{Histology}

The animals were sacrificed with an overdose of sodium pentobarbital $(100 \mathrm{mg} / \mathrm{kg})$ and perfused through the heart with physiological saline $(0.9 \% \mathrm{NaCl})$. The brains were removed and stored in formaldehyde (4\%) for a minimum of $24 \mathrm{~h}$. At least $48 \mathrm{~h}$ before the brains were sectioned, the brains were transferred to a $25 \%$ sucrose solution. The brains were sectioned $(60 \mu \mathrm{m})$ on a freezing microtome and mounted onto gelatin-subbed slides. The sections were then stained with thionin. The location of the infusion needles was determined by examining the sections under a microscope and using a rat brain atlas (Paxinos and Watson 2005). Only animals that had needle tips located within the BLA and had no lesions around the needle tips were included in the final analysis. As evidence indicates that there are differences between the anterior and posterior portions of the BLA (McDonald 1984), the A/P coordinates, in regard to Bregma, of 60 randomly selected rats (30 with left-side and 30 with right-side BLA cannulations) included in the final analysis were analyzed. The coordinates of left-side infusion sites were compared with those of right-side infusion sites.

\section{Statistics}

The retention latencies for Experiments 1 and 2 were analyzed with one-way analyses of variance (ANOVA). Fisher's post hoc tests were performed to determine the source of detected significances. An unpaired $t$-test was used to compare the A/P coordinates of infusion needle termination sites on the right and left sides. $P$ values of $<0.05$ were considered significant. All measures are expressed as mean \pm SEM. The number of animals in each group is indicated in the figure legends.

\section{Acknowledgments}

We thank Karen LeBorgne, Mylynn Nguyen, Michael Salama, Deena Ibrahim, Mallika Thomas, Matthew Sun, Brittney Holt, and David Seif for their excellent technical assistance. This research was supported by an NIH grant USPHS MH12526 (J.L.M.) and an NIH NRSA 1F31 MH073305-01 (R.T.L.).

\section{References}

Adamec, R.E. and Morgan, H.D. 1994. The effect of kindling of different nuclei in the left and right amygdala on anxiety in the rat. Physiol. Behav. 55: 1-12.

Andersen, S.L. and Teicher, M.H. 1999. Serotonin laterality in amygdala predicts performance in the elevated plus maze in rats. Neuroreport 10: 3497-3500. 
Baker, K.B. and Kim, J.J. 2004. Amygdalar lateralization in fear conditioning: Evidence for greater involvement of the right amygdala. Behav. Neurosci. 118: 15-23.

Cahill, L., Haier, R.J., White, N.S., Fallon, J., Kilpatrick, L., Lawrence, C., Potkin, S.G., and Alkire, M.T. 2001. Sex-related difference in amygdala activity during emotionally influenced memory storage. Neurobiol. Learn. Mem. 75: 1-9.

Cahill, L., Uncapher, M., Kilpatrick, L., Alkire, M.T., and Turner, J. 2004. Sex-related hemispheric lateralization of amygdala function in emotionally influenced memory: An fMRI investigation. Learn. Mem. 11: $261-266$.

Canli, T., Desmond, J.E., Zhao, Z., and Gabrieli, J.D. 2002. Sex differences in the neural basis of emotional memories. Proc. Natl. Acad. Sci. 99: 10789-10794.

Coleman-Mesches, K. and McGaugh, J.L. 1995. Differential effects of pretraining inactivation of the right or left amygdala on retention of inhibitory avoidance training. Behav. Neurosci. 109: 642-647.

Coleman-Mesches, K., Salinas, J.A., and McGaugh, J.L. 1996. Unilateral amygdala inactivation after training attenuates memory for reduced reward. Behav. Brain Res. 77: 175-180.

Ferry, B. and McGaugh, J.L. 1999. Clenbuterol administration into the basolateral amygdala post-training enhances retention in an inhibitory avoidance task. Neurobiol. Learn. Mem. 72: 8-12.

Hatfield, T. and McGaugh, J.L. 1999. Norepinephrine infused into the basolateral amygdala posttraining enhances retention in a spatial water maze task. Neurobiol. Learn. Mem. 71: 232-239.

Introini-Collison, I.B., Dalmaz, C., and McGaugh, J.L. 1996. Amygdala $\beta$-noradrenergic influences on memory storage involve cholinergic activation. Neurobiol. Learn. Mem. 65: 57-64.

Kebabian, J.W., Petzold, G.L., and Greengard, P. 1972. Dopamine-sensitive adenylate cyclase in caudate nucleus of rat brain, and its similarity to the "dopamine receptor". Proc. Natl. Acad. Sci.. 69: 2145-2149.

Kroner, S., Rosenkranz, J.A., Grace, A.A., and Barrionuevo, G. 2005. Dopamine modulates excitability of basolateral amygdala neurons in vitro. J. Neurophysiol. 93: 1598-1610.

LaLumiere, R.T., Buen, T.V., and McGaugh, J.L. 2003. Post-training intra-basolateral amygdala infusions of norepinephrine enhance consolidation of memory for contextual fear conditioning. J. Neurosci. 23: 6754-6758.

LaLumiere, R.T., Nguyen, L.T., and McGaugh, J.L. 2004. Post-training intrabasolateral amygdala infusions of dopamine modulate consolidation of inhibitory avoidance memory: Involvement of noradrenergic and cholinergic systems. Eur. J. Neurosci. 20: $2804-2810$.

LaLumiere, R.T., Nawar, E.M., and McGaugh, J.L. 2005. Modulation of memory consolidation by the basolateral amygdala or nucleus accumbens shell requires concurrent dopamine receptor activation in both brain regions. Learn. Mem. 12: 296-301.

Leonard, S.K., Anderson, C.M., Lachowicz, J.E., Schulz, D.W., Kilts, C.D., and Mailman, R.B. 2003. Amygdaloid D1 receptors are not linked to stimulation of adenylate cyclase. Synapse 50: 320-333.

Loretan, K., Bissiere, S., and Luthi, A. 2004. Dopaminergic modulation of spontaneous inhibitory network activity in the lateral amygdala Neuropharmacology 47: 631-639.

McDonald, A.J. 1984. Neuronal organization of the lateral and basolateral amygdaloid nuclei in the rat. J. Comp. Neurol. 222: 589-606.

McGaugh, J.L. 2004. The amygdala modulates the consolidation of memories of emotionally arousing experiences. Annu. Rev. Neurosci. 27: 1-28.

McGaugh, J.L., Introini-Collison, I.B., and Nagahara, A.H. 1988. Memory-enhancing effects of posttraining naloxone: Involvement of $\beta$-noradrenergic influences in the amygdaloid complex. Brain Res. 446: $37-49$.

Pare, D. and Collins, D.R. 2000. Neuronal correlates of fear in the lateral amygdala: Multiple extracellular recordings in conscious cats. $J$. Neurosci. 20: 2701-2710.

Paxinos, G. and Watson, C. 2005. The rat brain in stereotaxic coordinates. Elsevier Academic Press, Amsterdam.

Power, A.E., Roozendaal, B., and McGaugh, J.L. 2000. Glucocorticoid enhancement of memory consolidation in the rat is blocked by muscarinic receptor antagonism in the basolateral amygdala. Eur. J. Neurosci. 12: 3481-3487.

Power, A.E., McIntyre, C.K., Litmanovich, A., and McGaugh, J.L. 2003a. Cholinergic modulation of memory in the basolateral amygdala involves activation of both $\mathrm{m} 1$ and $\mathrm{m} 2$ receptors. Behav. Pharmacol. 14: 207-213.

. 2003b. Cholinergic modulation of memory in the basolateral amygdala involves activation of both $\mathrm{m} 1$ and $\mathrm{m} 2$ receptors. Behav. Pharmacol. 14: 207-213.

Quirarte, G.L., Roozendaal, B., and McGaugh, J.L. 1997. Glucocorticoid enhancement of memory storage involves noradrenergic activation in the basolateral amygdala. Proc. Natl. Acad. Sci. 94: 14048-14053.

Roozendaal, B., Quirarte, G.L., and McGaugh, J.L. 2002. Glucocorticoids interact with the basolateral amygdala $\beta$-adrenoceptor-camp/ camp/pka system in influencing memory consolidation. Eur. J. Neurosci. 15: 553-560.

Rosen, G.D., Finklestein, S., Stoll, A.L., Yutzey, D.A., and Denenberg, V.H. 1984. Neurochemical asymmetries in the albino rat's cortex, striatum, and nucleus accumbens. Life Sci. 34: 1143-1148.

Rosenkranz, J.A. and Grace, A.A. 2002. Cellular mechanisms of infralimbic and prelimbic prefrontal cortical inhibition and dopaminergic modulation of basolateral amygdala neurons in vivo. J. Neurosci. 22: 324-337.

Wilensky, A.E., Schafe, G.E., and LeDoux, J.E. 2000. The amygdala modulates memory consolidation of fear-motivated inhibitory avoidance learning but not classical fear conditioning. J. Neurosci. 20: 7059-7066.

Yajeya, J., de la Fuente Juan, A., Bajo, V.M., Riolobos, A.S., Heredia, M. and Criado, J.M. 1999. Muscarinic activation of a non-selective cationic conductance in pyramidal neurons in rat basolateral amygdala. Neuroscience 88: 159-167.

Received April 28, 2005; accepted in revised form July 20, 2005.
532 Learning \& Memory

www.learnmem.org 

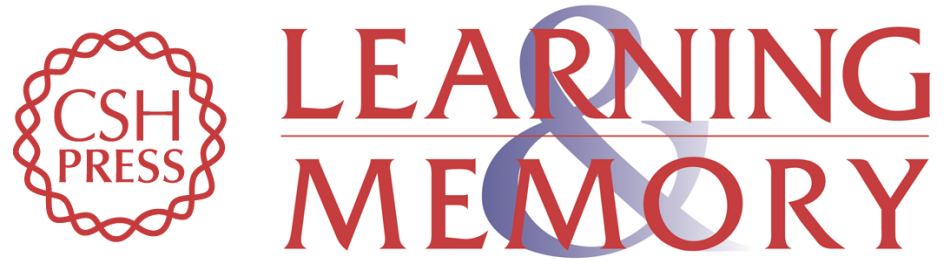

\section{Memory enhancement induced by post-training intrabasolateral amygdala infusions of $\beta$-adrenergic or muscarinic agonists requires activation of dopamine receptors: Involvement of right, but not left, basolateral amygdala}

Ryan T. LaLumiere and James L. McGaugh

Learn. Mem. 2005, 12:

Access the most recent version at doi:10.1101//m.97405

References

Email Alerting Service

\section{License}

This article cites 30 articles, 9 of which can be accessed free at: http://learnmem.cshlp.org/content/12/5/527.full.html\#ref-list-1

Receive free email alerts when new articles cite this article - sign up in the box at the top right corner of the article or click here. 